\title{
Meson Photoproduction (CLAS)
}

- Spectroscopy of baryons

- Spectroscopy of mesons

- $\rho$-mesons in the medium

- Reaction mechanisms

Steffen Strauch (University of South Carolina)

for the CLAS Collaboration

XVII International Workshop on Deep-Inelastic Scattering and Related Subjects DIS 2009

26-30 April 2009, Madrid 


\section{The CEBAF Large Acceptance Spectrometer}

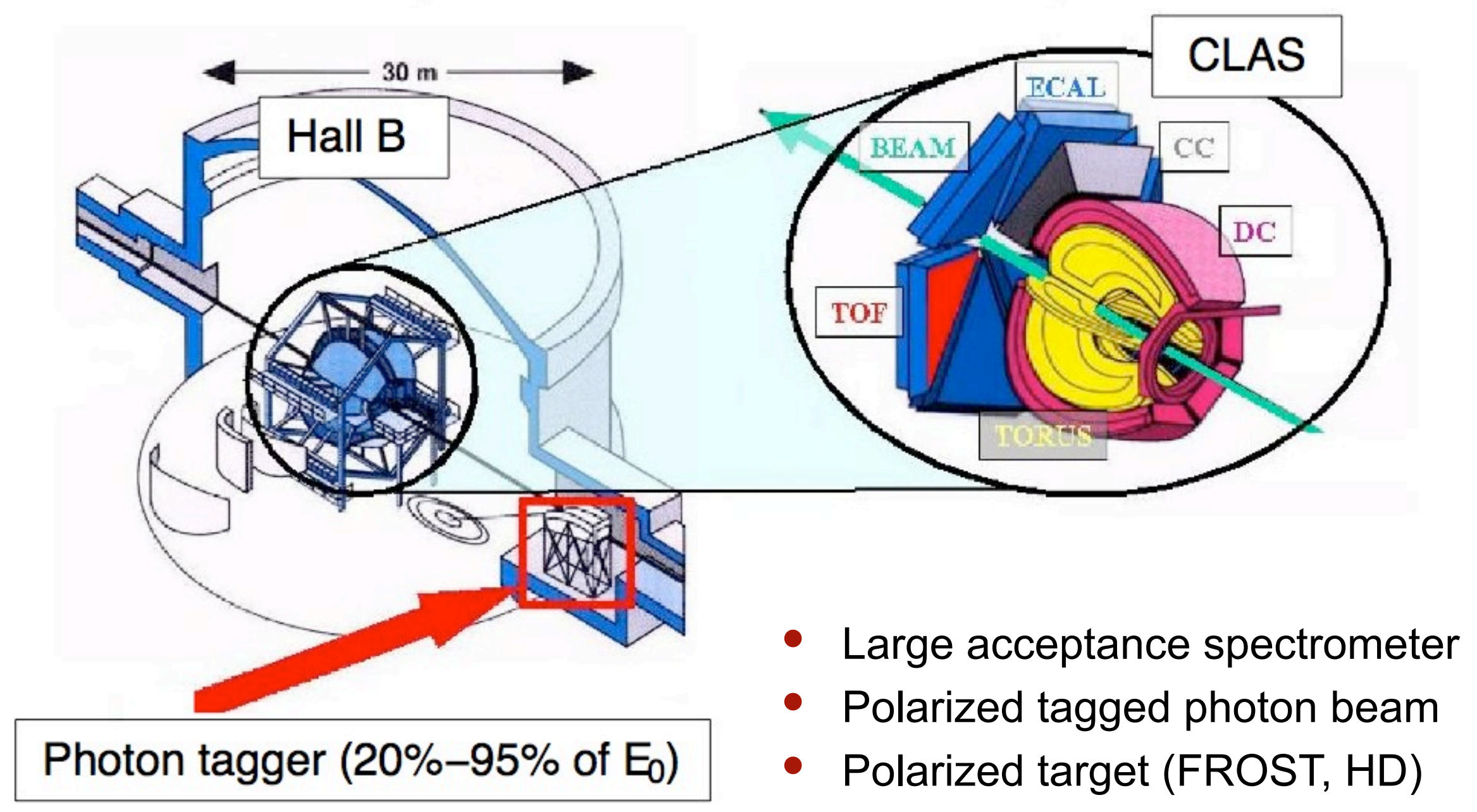




\section{Studying the Excited States of the Nucleon}

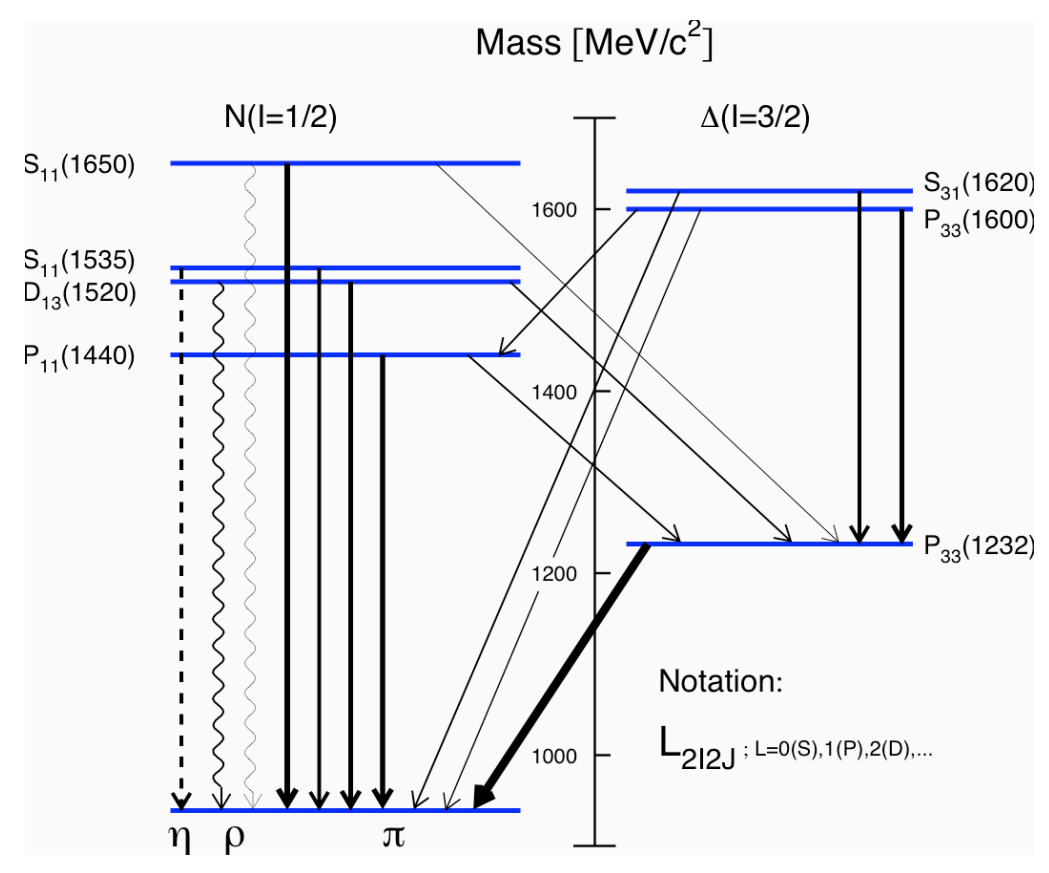

$\gamma N \rightarrow N \pi, N \pi \pi, N \eta, Y K, \ldots$
- The location and properties of excited states reflect the dynamics and relevant degrees-of-freedom within the nucleons.

\section{Quark Models}

- Symmetric Constituent Quark Models predict overabundance of excited states ("missing" resonance problem)

- Quark-Diquark Models predict fewer states

- Quark and Flux-Tube Models predict increased number of states

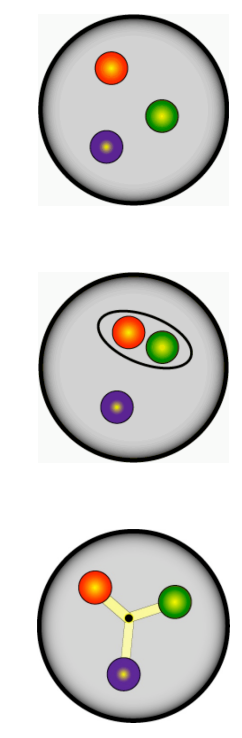

3 


\section{Extraction of Resonance Parameters}

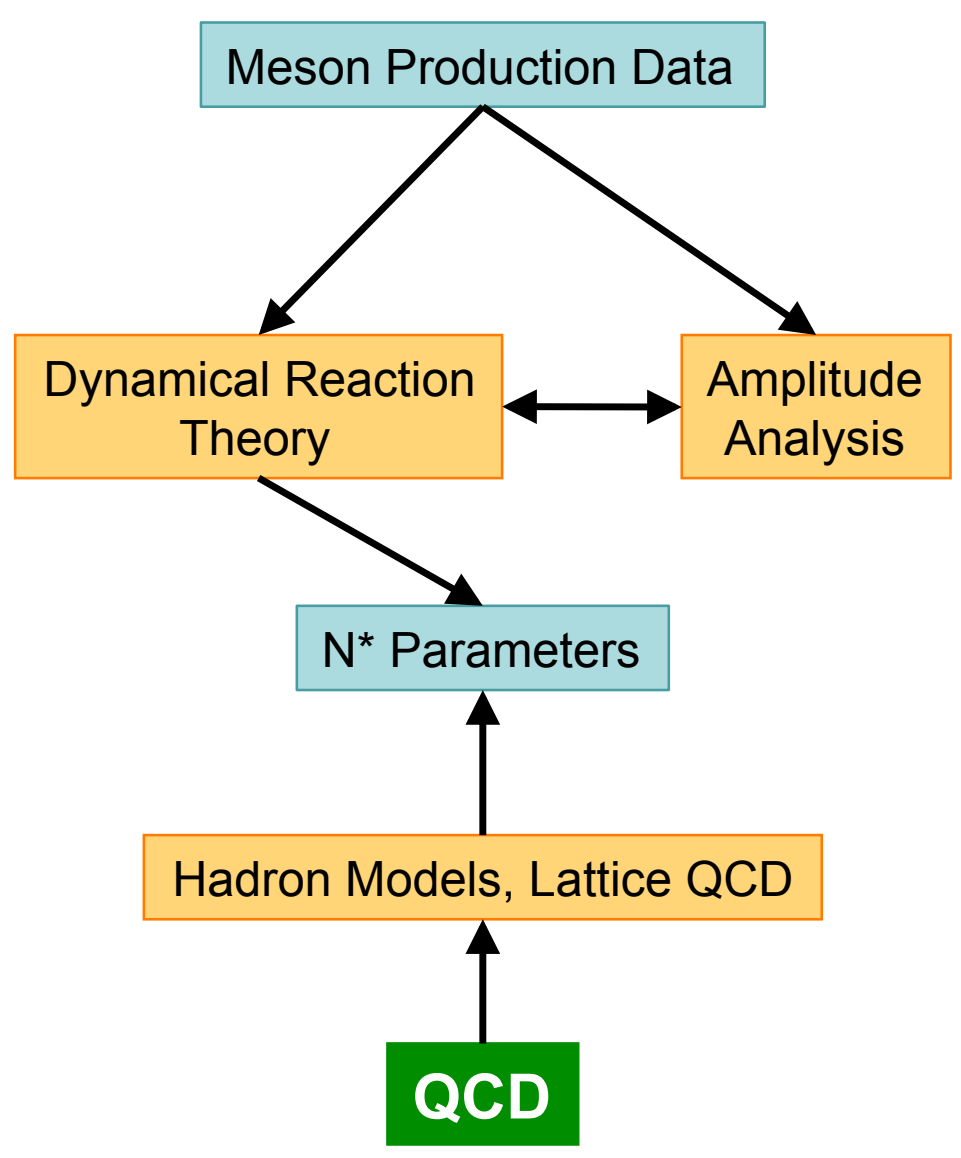

- Measurement of eight observables needed to unambiguously determine the four amplitudes of single meson photoproduction:

- differential cross section: $d \sigma / d \Omega$

- single polarization observables: $\mathrm{P}, \mathrm{T}, \Sigma$

- double polarization observables

- CLAS experiments with

- polarized beam

- polarized target (FROST, HD)

- recoil polarization (weak decay of hyperons) 


\section{$\pi^{+}$Photoproduction on the Proton}

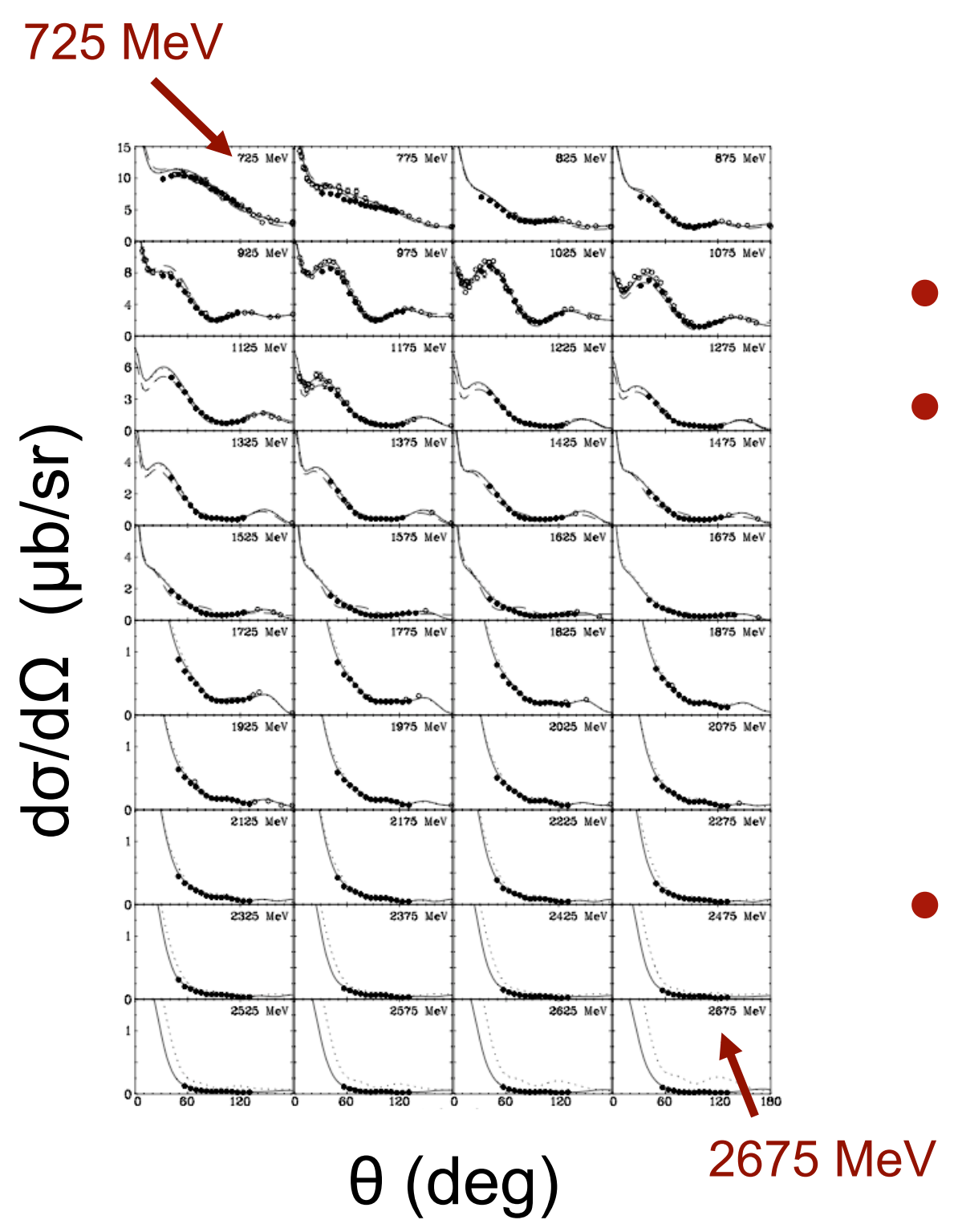

$$
\gamma p \rightarrow \pi^{+} n
$$

- $\pi^{+} \mathrm{n}$ data up to $2675 \mathrm{MeV}$

- SAID multipole analysis:

- significant new constraints at high energies

- no significant change of resonance parameters

- need measurements of spin observables

M. Dugger et al., arXiv:0903.1110v1 [hep-ex] 


\section{Hyperon Recoil Polarization P}

$a^{4}$
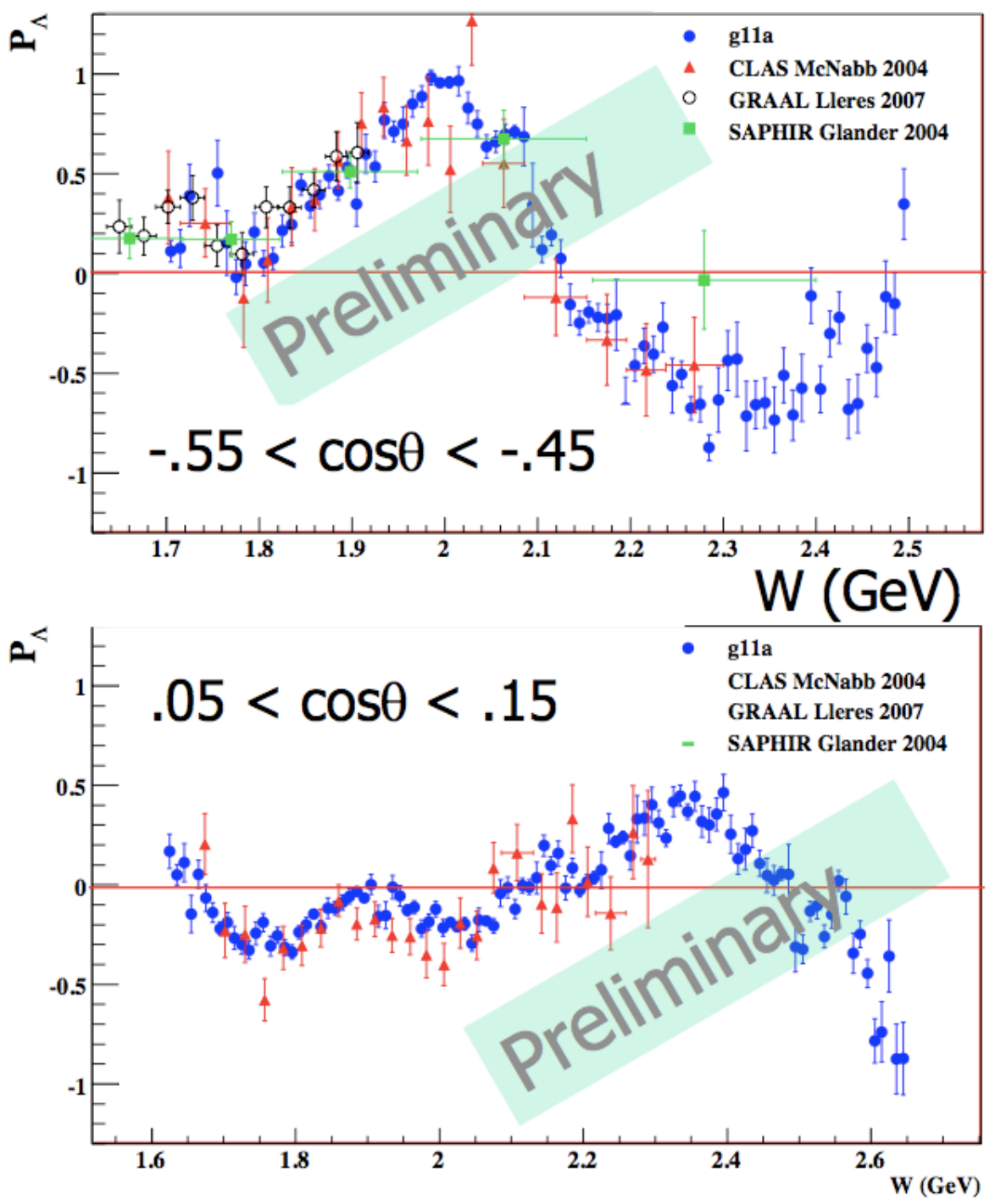

$$
\gamma p \rightarrow K^{+} \vec{\Lambda}
$$

- Induced polarization $\mathrm{P}_{\wedge}$

- Preliminary CLAS'09 data has best coverage yet

- Good agreement with previous data

- CLAS PWA in progress

Figures from: R. Schumacher, NSTAR 2009; M. McCracken (CMU) Ph.D. work; J. McNabb et al. Phys. Rev. C 69, 043302 (2004). 


\section{Polarization Transfer Observables $C_{x}, C_{z}$}

$\mathrm{C}_{\mathrm{x}}(\bullet), \mathrm{C}_{\mathrm{z}}(\mathrm{O})$ for $\mathrm{K}^{+} \wedge$ channel

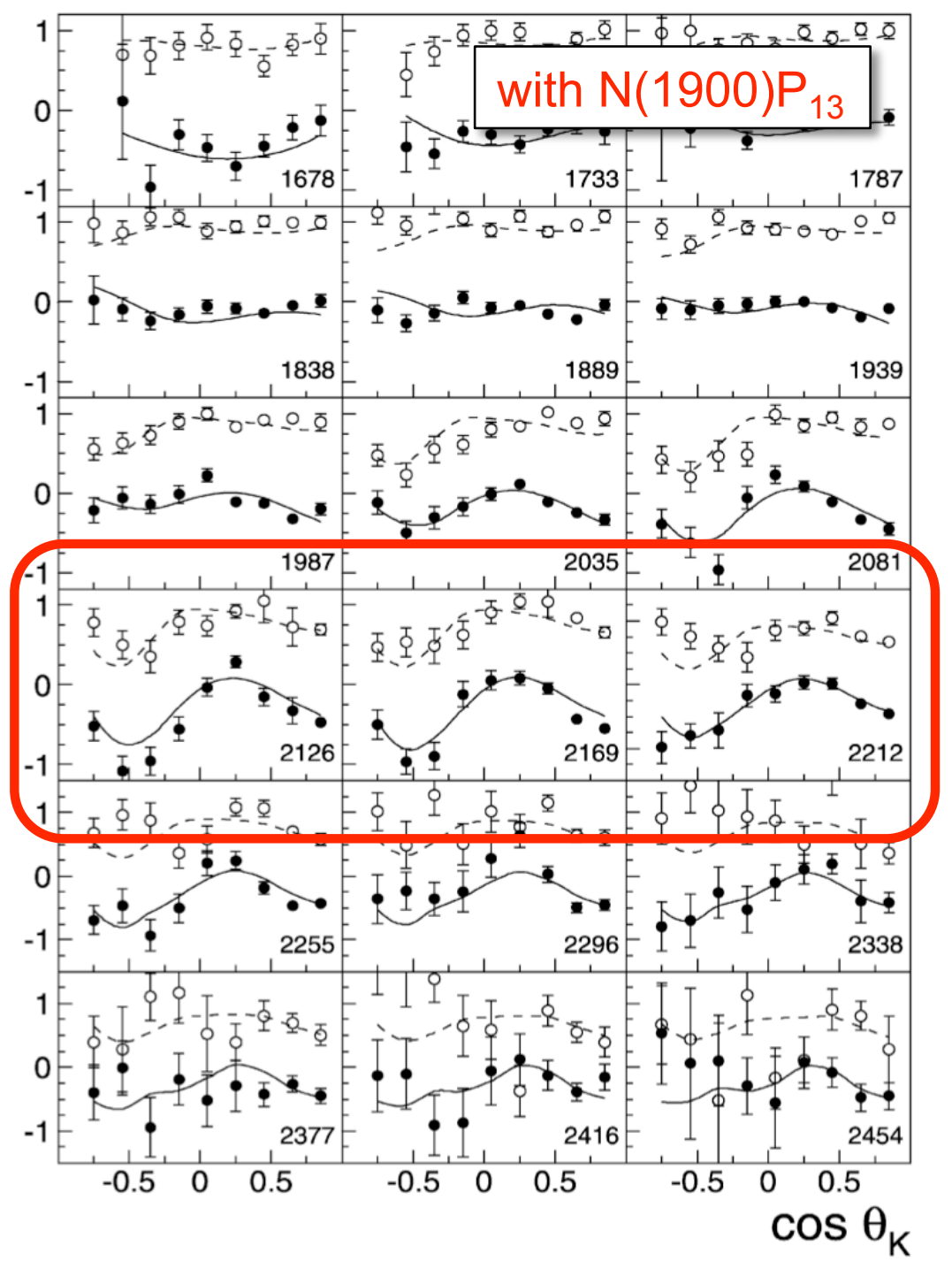

$$
\vec{\gamma} p \rightarrow K^{+} \vec{\Lambda}
$$

- $\mathrm{N}(1900) \mathrm{P}_{13}$ needed in PWA of Nikonov et al.

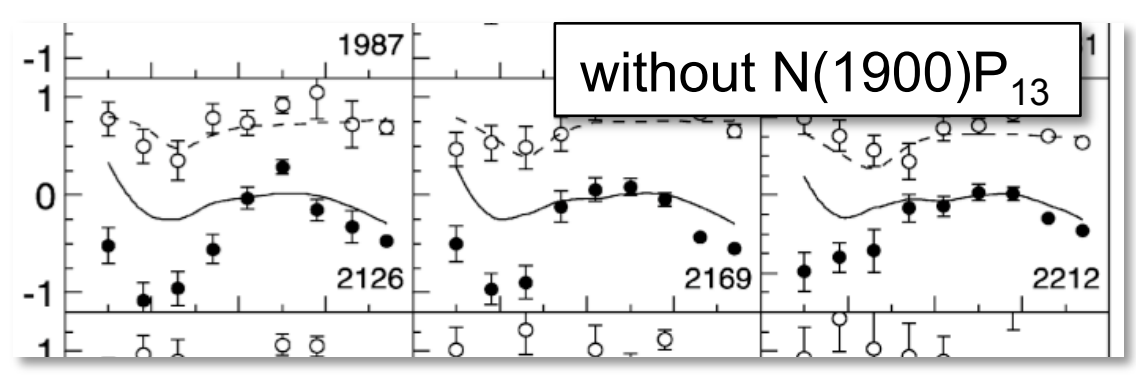

- $N(1900) P_{13}$ found in qqq models, not in quark-diquark models.

\section{Data: R. Bradford, et al., Phys. Rev. C 75, 035205 (2007). Analysis: V.A. Nikonov et al., Phys. Lett. B 662, 245 (2008)




\section{Beam-Helicity Asymmetries in Double-Pion Photoproduction on the Proton}

- Dominant nucleon-resonance decay channels $W>1.6 \mathrm{GeV}: \quad \pi \pi N$
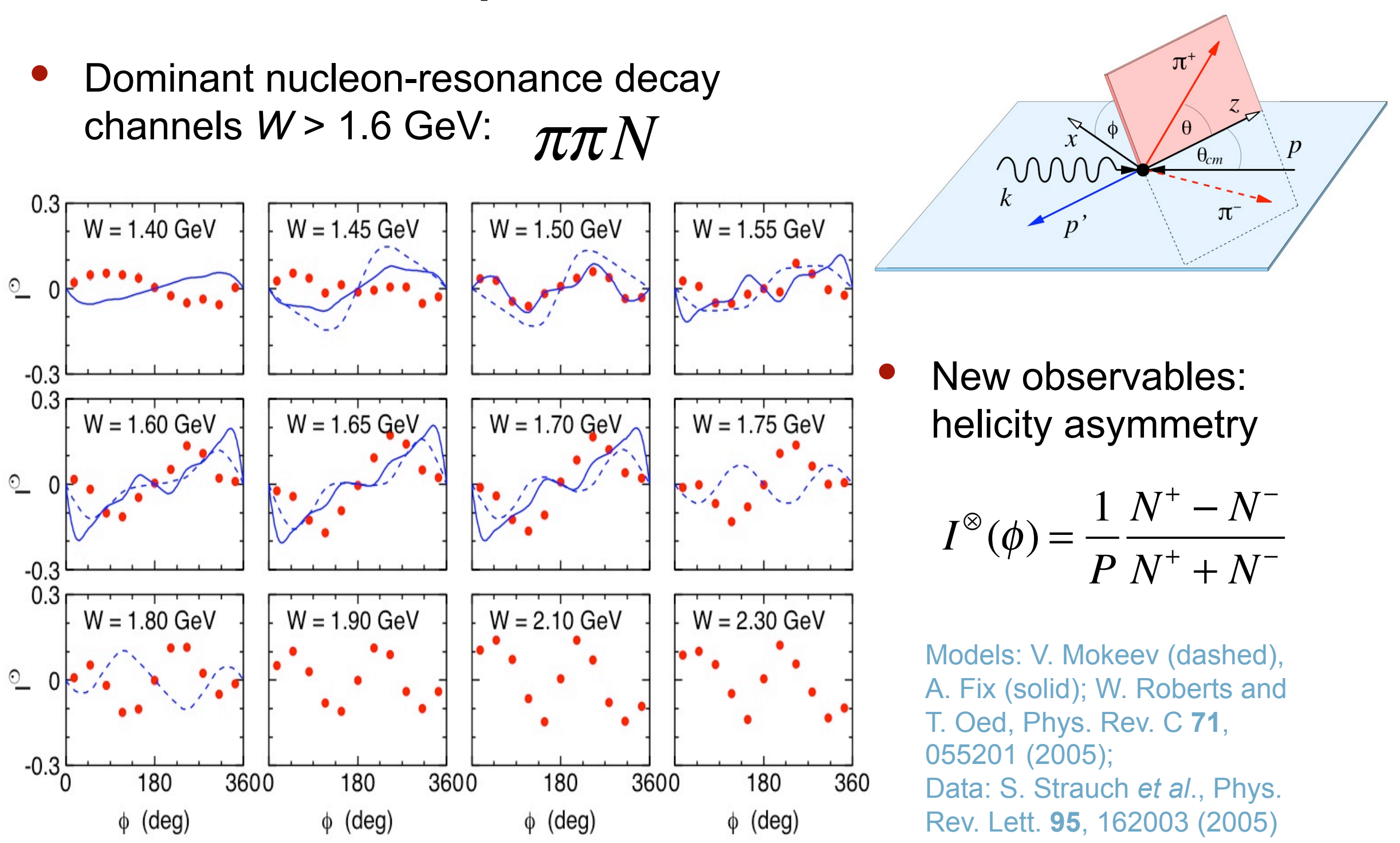

- New observables: helicity asymmetry

$$
I^{\otimes}(\phi)=\frac{1}{P} \frac{N^{+}-N^{-}}{N^{+}+N^{-}}
$$
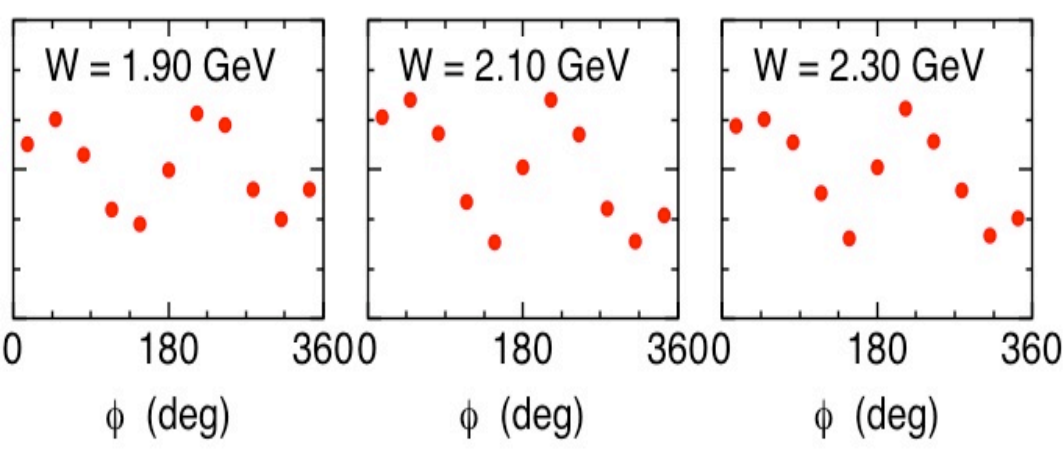

Models: V. Mokeev (dashed),

A. Fix (solid); W. Roberts and

T. Oed, Phys. Rev. C 71, 055201 (2005);

Data: S. Strauch et al., Phys. Rev. Lett. 95, 162003 (2005) 


\section{Sequential Decay}

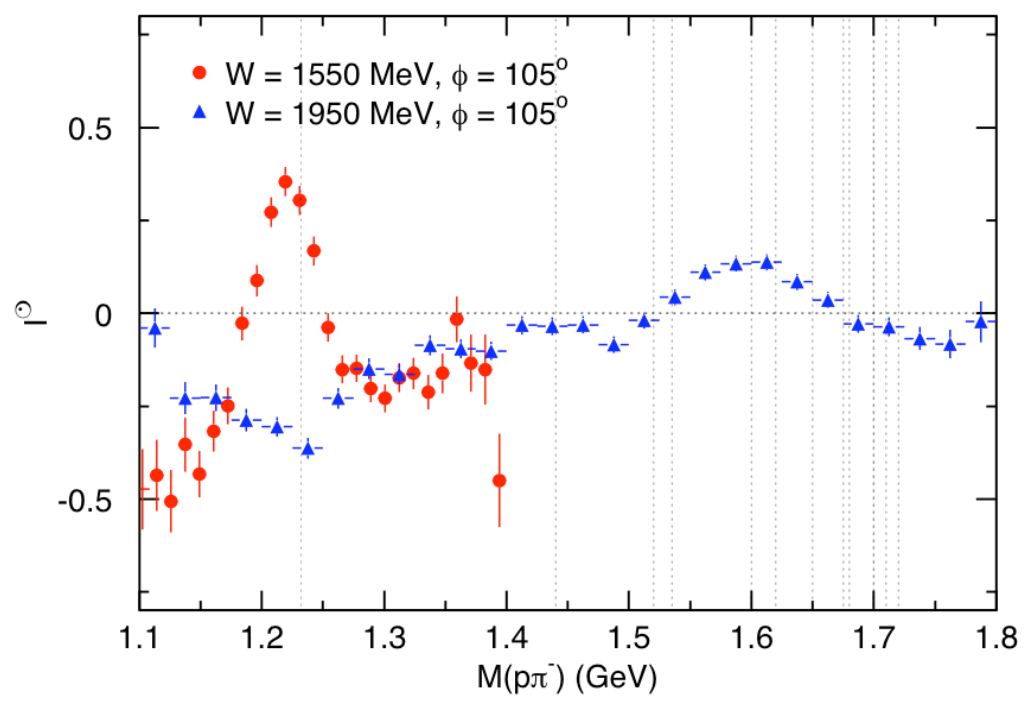

- $\mathrm{\gamma N} \rightarrow \mathrm{N}^{*} \rightarrow \pi \Delta \rightarrow \mathrm{\pi} \mathrm{m} \mathrm{N}$

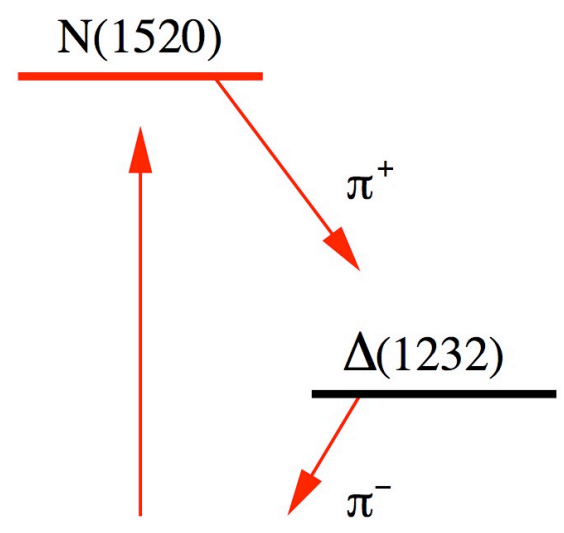

$\mathrm{p}=\mathrm{N}(938)$

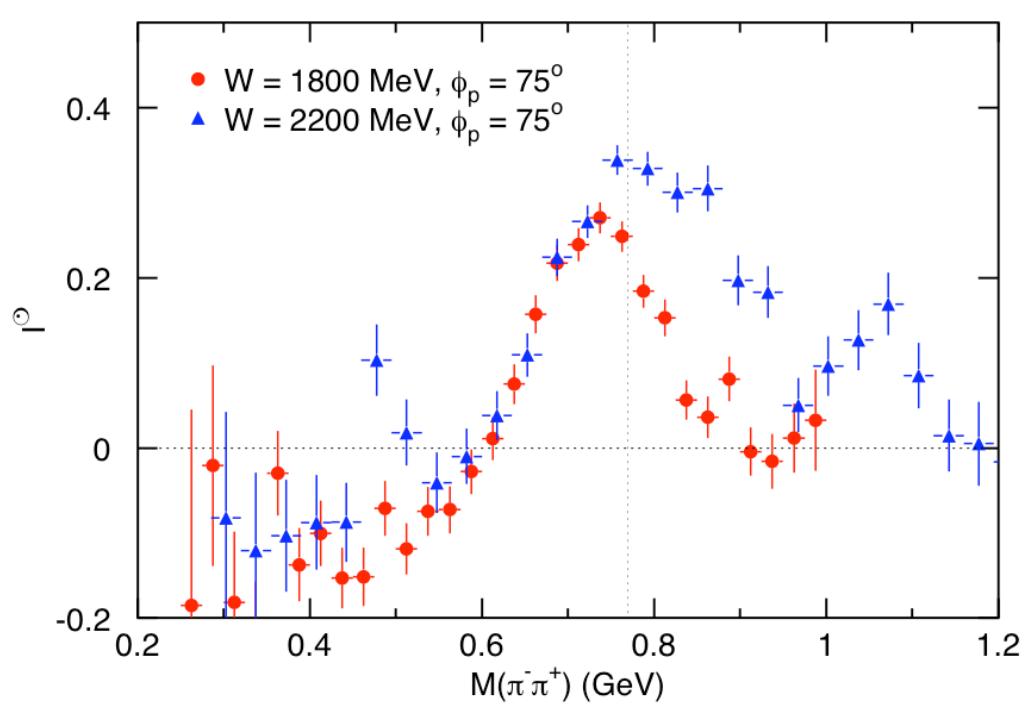

- $\gamma \mathrm{N} \rightarrow \rho \mathrm{N} \rightarrow \pi \pi \mathrm{N}$

- Helicity asymmetries allow detailed study of the $\pi N \rightarrow$ $\pi т N$ reaction (e.g., sequential decay)

- Extension of these studies in E06-013 (FROST). 


\section{Measurement of Direct $f_{0}(980)$ Photoproduction on the Proton}

Moments of dipion angular distribution
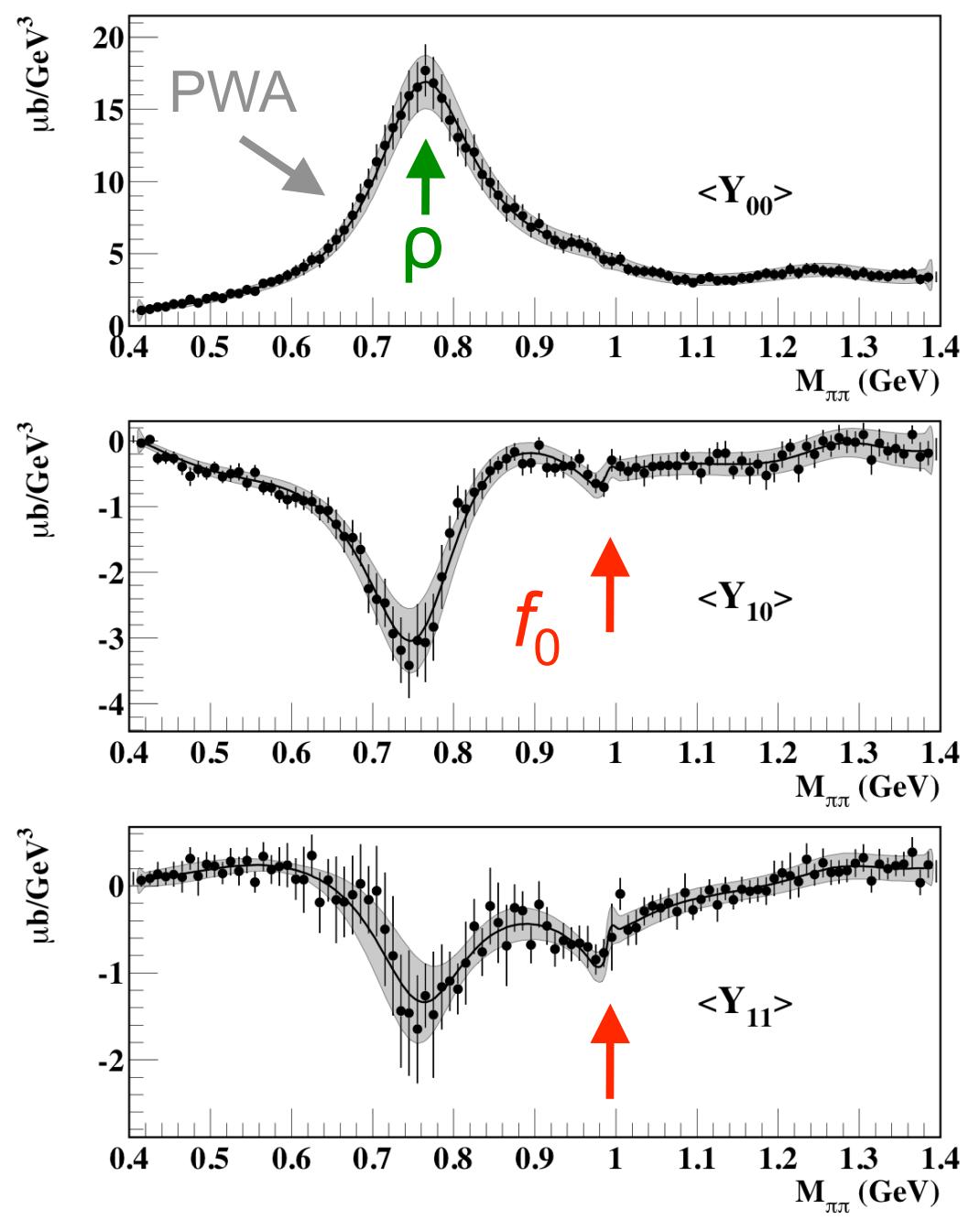

M. Battaglieri et al., Phys. Rev. Lett. 102, 102001 (2009) $\gamma p \rightarrow p \pi^{+} \pi^{-}$

- Low lying scalar mesons: quark-antiquark or diquarkantidiquark bound states?
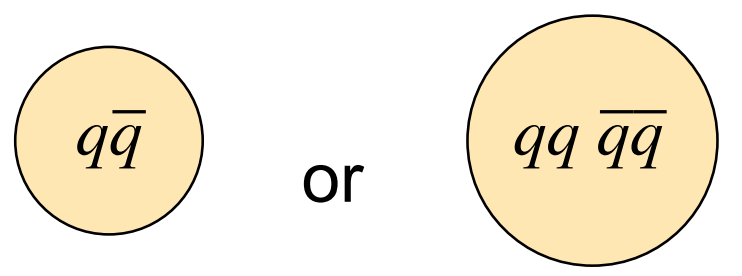

- Interference between $P$ and $S$ waves at $M_{\pi \pi} \sim 1 \mathrm{GeV}$ indicates presence of $f_{0}(980)$ resonance (first time in a photoproduction experiment). 


\section{Search for the Photoexcitation of Exotic Mesons in the $\pi^{+} \pi^{+} \pi^{-}$System}

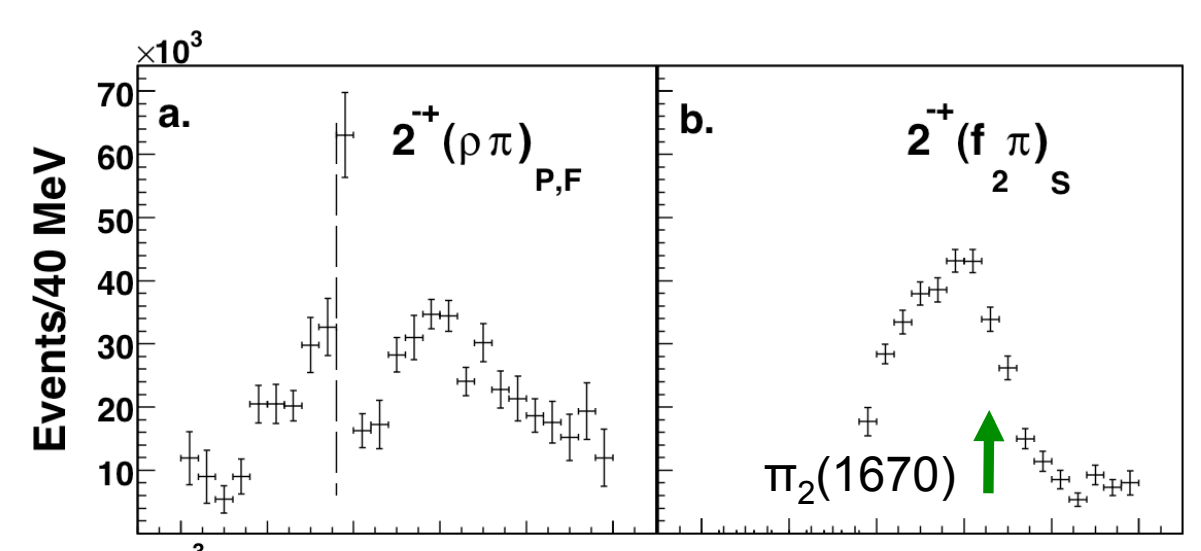

$$
\gamma p \rightarrow \pi^{+} \pi^{+} \pi^{-}(n)
$$

- Lowest lying $(\bar{q} q g)$ state predicted to have $\mathrm{JPC}^{\mathrm{PC}}=1^{-+}$
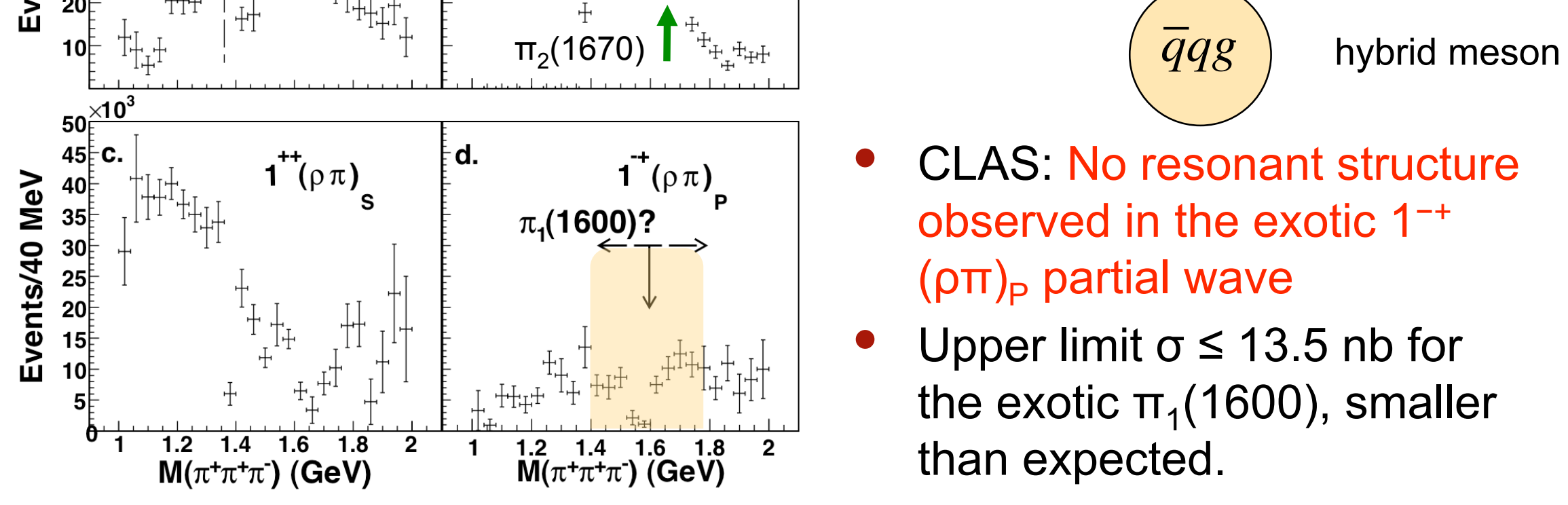

- CLAS: No resonant structure observed in the exotic $1^{-+}$ $(\rho \pi)_{\mathrm{P}}$ partial wave

- Upper limit $\sigma \leq 13.5 \mathrm{nb}$ for the exotic $\pi_{1}(1600)$, smaller than expected.

- Calculated photo-production cross section overestimated or $\pi_{1}(1600)$ not exotic? 


\section{In-Medium Properties of Vector Mesons}

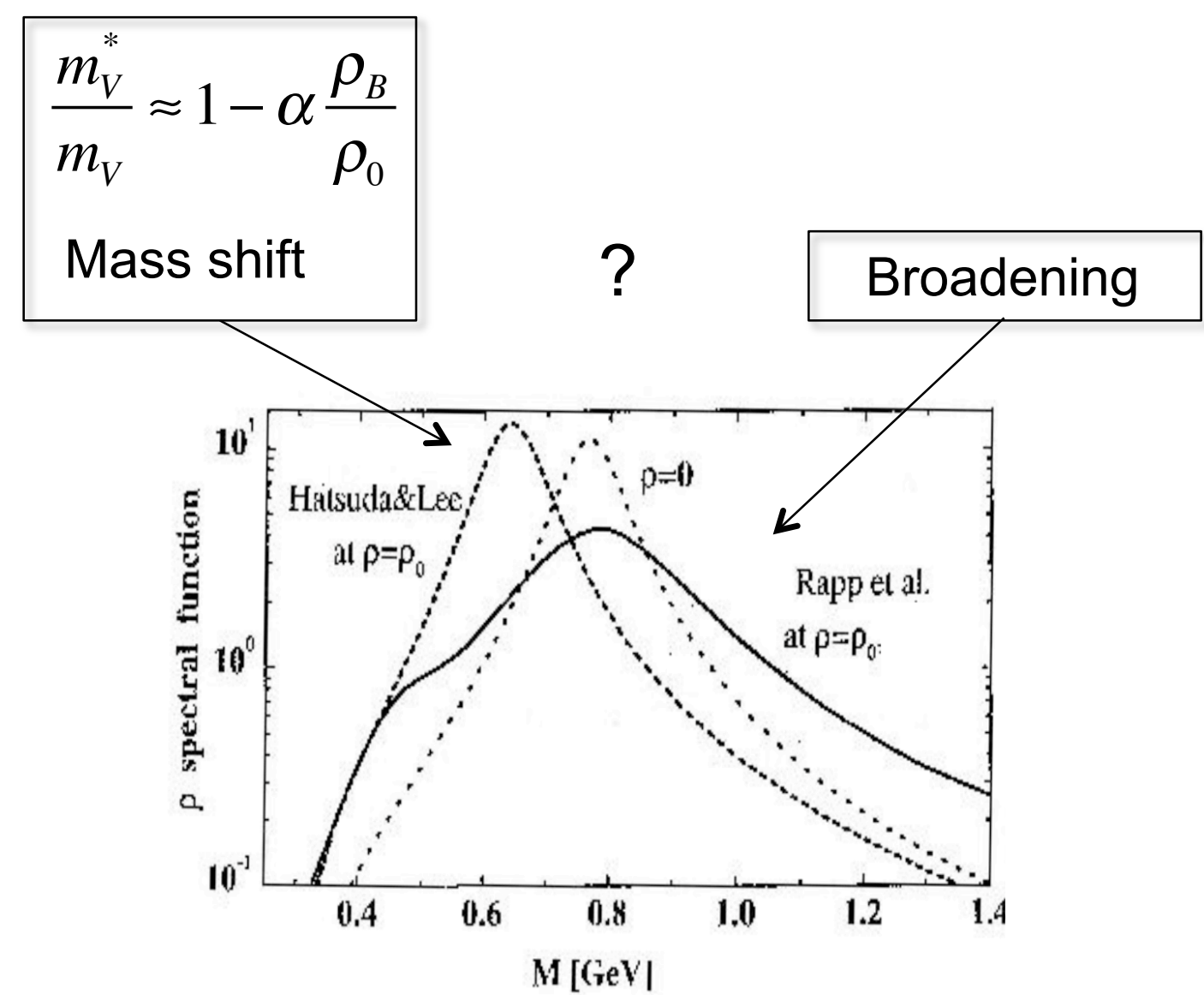

$\rho$-meson spectral function (possibly momentum dependent)
- The dynamical breaking of chiral symmetry has large impact on the masses of hadrons.

- Restoration of chiral symmetry in hot and/or dense nuclear matter is predicted.

$\Rightarrow$ expected changes of in-medium properties of hadrons 


\section{Medium Modification of Vector Mesons}

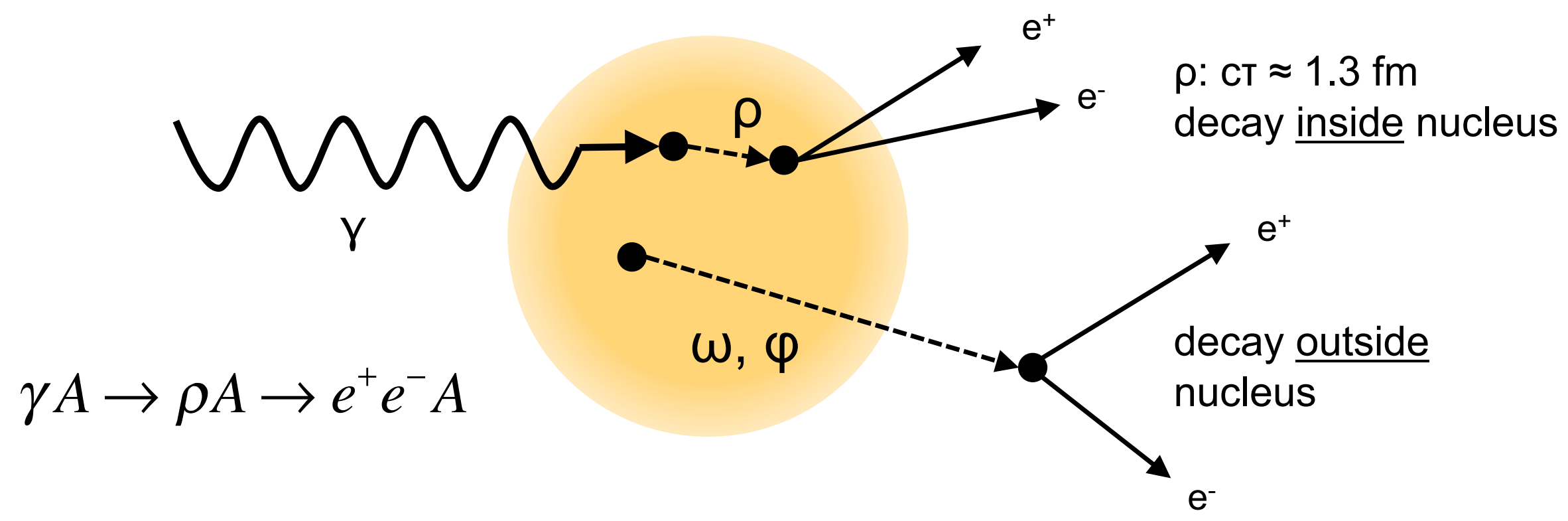

- CLAS g7a Experiment (medium modification at $T=0$ ):

- Targets: LD2, C, Ti, Fe, (Pb)

- Leptonic decay with almost no final state interaction; $\Gamma_{\mathrm{e}+\mathrm{e}} / \Gamma_{\text {tot }} \sim 5 \times 10^{-5}$

- Momentum of $\rho$ between 0.8 and $2 \mathrm{GeV}$ (>0.5 GeV with g7b)

- Study invariant mass distribution, $\mathrm{m}\left(\mathrm{e}^{+} \mathrm{e}^{-}\right)$ 


\section{The p-Mass Spectra}

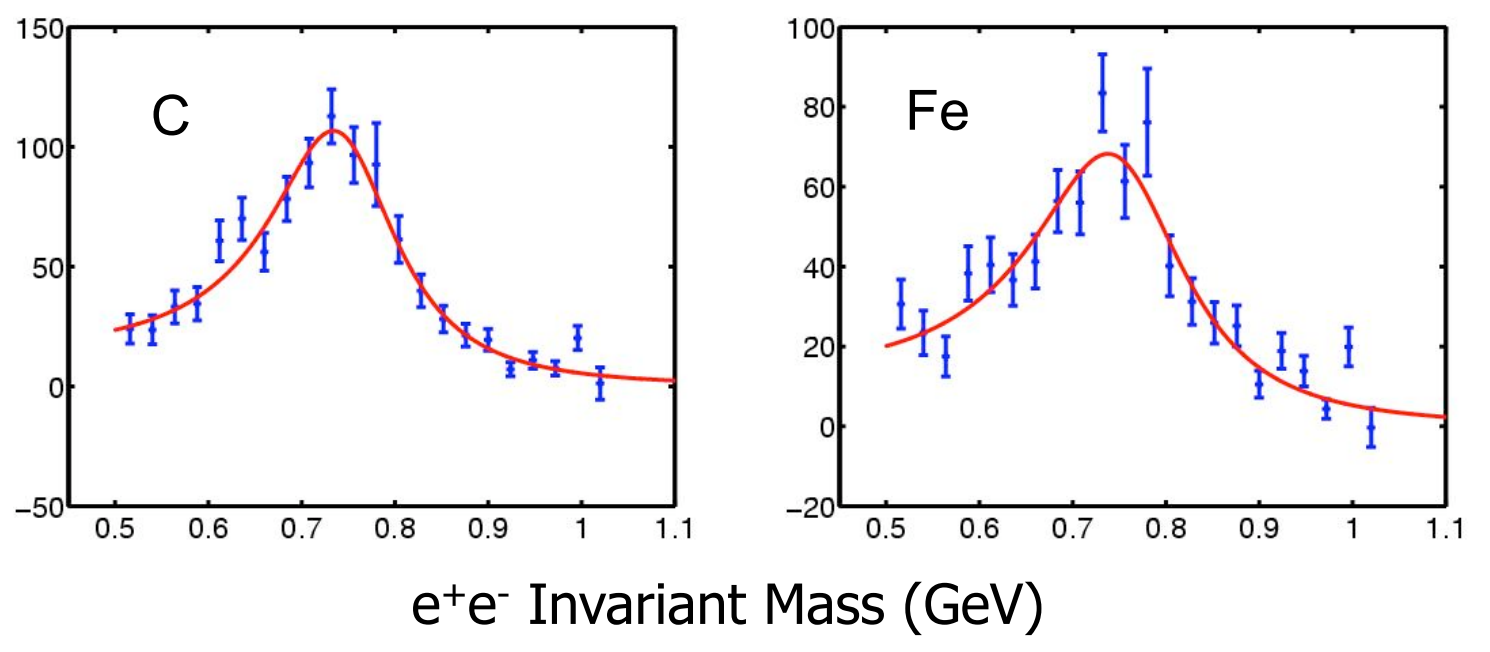

Extraction of $\rho$-mass spectra after subtraction of $\omega$ and $\varphi$ contributions and combinatorial background.

\begin{tabular}{|l|l|l|l|l|}
\hline Target & $\begin{array}{l}\text { Mass }\left(\mathrm{MeV} / \mathrm{c}^{2}\right) \\
\text { CLAS data }\end{array}$ & $\begin{array}{l}\text { Width }\left(\mathrm{MeV} / \mathrm{c}^{2}\right) \\
\text { CLAS data }\end{array}$ & $\begin{array}{l}\text { Mass }\left(\mathrm{MeV} / \mathrm{c}^{2}\right) \\
\text { Giessen BUU }\end{array}$ & $\begin{array}{l}\text { Width }\left(\mathrm{MeV} / \mathrm{c}^{2}\right) \\
\text { Giessen BUU }\end{array}$ \\
\hline${ }^{12} \mathrm{C}$ & $762.5 \pm 3.7$ & $176.4 \pm 9.5$ & $773.8 \pm 0.9$ & $177.6 \pm 2.1$ \\
\hline${ }^{48} \mathrm{Ti}-56 \mathrm{Fe}$ & $779.0 \pm 5.7$ & $217.7 \pm 14.5$ & $773.8 \pm 5.4$ & $202.5 \pm 11.6$ \\
\hline
\end{tabular}

- The vacuum properties of the $\rho$ meson are: $m=770 \mathrm{MeV} / \mathrm{c}^{2}$ and $\Gamma=150 \mathrm{MeV}$.

- No mass shift; broadening of the width is consistent with many-body effects. 


\section{$\phi(1020)$ Meson Photoproduction}

- Photoproduction of $\phi$-mesons on the proton at large momentum transfer

E. Anciant et al., Phys. Rev. Lett. 85, 4682 (2000)

- Tensor polarization of the $\phi$-meson photoproduced at high $\mathrm{t}$

K. McCormick et al., Phys. Rev. C 69, 032203(R) (2004)

- Measurement of coherent $\phi$-meson photoproduction from the deuteron at low energies

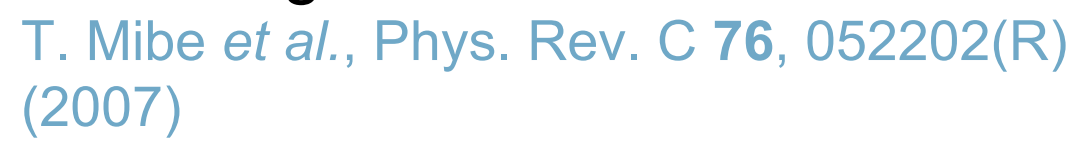

- Extensive analysis of $\phi$-meson

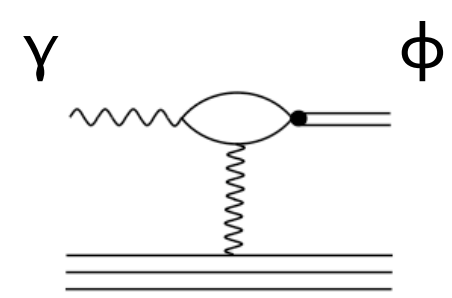

$\phi$

\section{Pomeron}
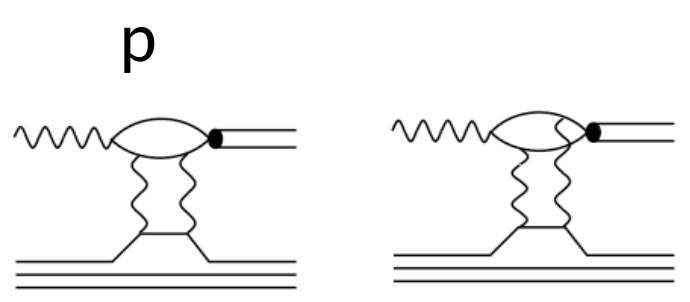

2 gluons photoproduction from g11 data
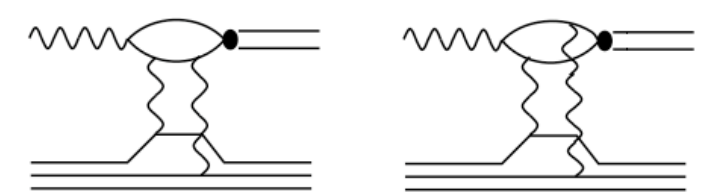

correlations

D. Tedeschi, Diffraction 2008 


\section{Summary}

- Spectroscopy of baryons

- high precision unpolarized and polarized photoproduction data with various decay channels, covering large angular and energy ranges provide needed input for partial wave analyses in the search for new baryon states

- Spectroscopy of mesons

- $\rho$-mesons in the medium

- no significant mass shift observed; width consistent with expected collisional broadening

- Reaction mechanisms 